\title{
Editorial
}

The first issue of Thorax was published 32 years ago. Until recently, the journal was owned by the British Medical Association but its contents and editorial policy were controlled by the Thoracic Society. From the beginning of this year, as a result of a new agreement, Thorax will be owned jointly by the British Medical Association and the Thoracic Society.

We hope that our readers will take pleasure in the newly designed cover, the first change in external appearance since the journal's inception. Our changes are not limited to the outward appearance of Thorax. The Editorial Board has been enlarged to achieve or augment representation in many specialist branches of thoracic medicine and surgery although the editors will also continue to appoint other prominent experts to assess the work of contributors.

Beginning with this issue, we shall publish regular editorials on topical or controversial subjects. We hope that these will disentangle matters of confusion, question strongholds of dogmatism, and sometimes express an individual and eccentric point of view. Thorax will also publish correspondence concerning our original contributions and will print authors' replies to comments on and criticism of their work. A lively correspondence column adds spice to the meat proffered by the original papers and, we hope, will stimulate further discussion. On page 10 is the first of a series of articles on the historical aspects of chest medicine. We should like to think that these will have a particular appeal to our younger readers, who might wish to acquire a sense of historical perspective in relation to the study of chest disease.

Until now it has been necessary to reject a high proportion of the isolated case reports submitted to us, solely because of lack of space. Many of these deserve publication, either because the condition has not previously been described, or because they reveal an unusual pattern of behaviour in an otherwise well-known disease. We hope to publish a larger selection of these case reports by offering to authors the alternative of publication as 'short reports' limited to 600 words. Details appear on the inside front cover.

Thorax publishes original work in every field of chest medicine and the related basic sciences. A review of the subjects covered in recent years has shown a deficiency of papers on anaesthesia, microbiology, radiology, and industrial medicine. We should like to encourage original work in these important specialties. Contributions on these subjects will be welcomed and given special consideration.

Thorax has always maintained a high standard in the content of its original contributions and in the quality and number of its radiographs and photomicrographs. The editors will continue to follow the ideals of excellence established by their predecessors and in these respects Thorax has not changed.

BENJAMIN B. MILSTEIN ANTHONY SEATON

\section{Asthma-contrasts in care}

It may be estimated that at any time approximately $2 \%$ of the population are suffering from asthma, and that in Britain two in every thousand asthmatics die of the disease each year. Asthma afflicts people of all ages and, while it is easily recognised in young non-smokers, it is frequently misdiagnosed as bronchitis or emphysema among the elderly. It is a frequent experience for chest physicians to see patients who have been disabled by years of breathlessness and yet whose whole life may be improved dramatically by simple treatment. Even when asthma has been correctly diagnosed, it is still commonplace to see the patient treated with old, often potentially addictive, barbiturate-containing drugs without attempts to monitor their efficacy and in a haphazard and arbitrary manner. Patients are still warned not to overuse their aerosol bronchodilators but are given no advice on what to do if these become ineffective. Severe exacerbations of the disease are frequently treated with a change of bronchodilator rather than by the introduction of the necessary corticosteroids.

Any doctor working in an asthma clinic is quickly impressed by the number of misconceptions in the minds of his colleagues about the 
disease. The importance of psychological factors is frequently stressed in referral letters, and patients are usually treated with sedative drugs. Yet it is remarkable how quickly the anxiety or depression melt away when the patient finds a doctor who realises that its primary cause is the fear of death in an attack rather than vice versa. The overprotective, anxious mother suddenly becomes normal again when her child stops waking in the night with terrifying attacks and is no longer too tired in the morning to go to school. Patients are often referred for 'desensitisation' when their attacks are provoked by everything from colds to exertion and when there is scant evidence that any presently used technique is of any value. Children are prevented from playing games rather than given cromoglycate, and steroids are withheld or withdrawn from patients because of their potential danger without the actual danger to the patient from his asthma being assessed by measurement of his peak flow.

In almost no other field is the gap between diagnostic and therapeutic knowledge and its general application so great. It is hard to understand why this should be so as the principles of diagnosis - to demonstrate reversibility of airways obstruction-and of treatment-to reverse the obstruction by one or more of only three types of drug-are so simple. Even though the fundamental biochemical and immunological mechanisms of the disease remain ill-understood, the management of asthma both from day to day and in acute severe attacks has been well studied and in many aspects may be based on the results of properly conducted controlled trials. Most new drugs introduced for the management of asthma may also be used in the knowledge that their efficacy has been convincingly demonstrated. The blame for this failure of communication must rest with hospital physicians, who have been so slow to adapt them- selves to changes in the understanding of a common disease, and with epidemiologists, who have successfully publicised the dubious toxicity of our most useful remedies without pointing out the therapeutic alternatives.

It is interesting to contrast the management of asthma with that of diabetes, another chronic disease punctuated by life-threatening episodes. How many physicians would manage diabetes without measuring the blood and urine sugar levels? Yet these same physicians usually feel able to look after severe asthmatics without ever measuring their peak flow rate or arterial blood gases. The analogy with diabetes may be taken further. In both diseases, proper management depends on education of the patient to understand his condition, to recognise signs of deterioration, and to adjust his own treatment accordingly. In many cases the patients find they know more about their disease than the doctor, a situation that the less flexible among us find difficult to tolerate. Patients with these diseases seem prepared to attend busy special clinics where they can discuss any problems with and receive advice from a physician who has much experience of the condition. However, there are too many asthmatics to be catered for by such clinics, and the proper place for their long-term care is in general practice. Only when all physicians and general practitioners equip themselves with a cheap device for measuring peak flow and with a basic knowledge of modern asthma therapy will life become more bearable for the majority of asthmatics and will unnecessary deaths be prevented. The major problem in asthma today is the education of the profession about the disease.

ANTHONY SEATON Asthma Research Unit, Sully Hospital, Penarth, Glam. 